\title{
Bosques andinos del sur de Ecuador, clasificación, regeneración y uso
}

\section{Andean Forests of Southern Ecuador, classification, regeneration and use}

\author{
Rainer W. Bussmann*
}

Publicación: 08/12/2005

\section{Resumen}

Existen muy pocos inventarios y descripciones sintaxonómicas de la vegetación de bosques montanos tropicales. Este trabajo presenta datos sobre los bosques del sur de Ecuador localizados en la Reserva Biológica San Francisco, límite norte del Parque Nacional Podocarpus. Se propone la siguiente clasificación junto con notas sobre los factores edáficos que contribuyen a su establecimiento. El «Bosque Montano Bajo» (1800-2150 m), formando el orden Alzateetalia verticillatae, muestra 2-3 estratos arbóreos muy diversos, con árboles de 20-35 m de altura, y es una clímax mosaico típico. Creciendo sobre Terric Haplosaprists y Aquic Dystrupepts, originado del material de derrumbes viejos, este tipo de bosque se extiende hasta los $2300 \mathrm{~m}$ sobre el nivel del mar en quebradas protegidas de viento. A altitudes de $2100-2650 \mathrm{~m}$ (menos de $2750 \mathrm{~m}$ ) la estructura del bosque y la composición florística cambia totalmente. Esta vegetación, el «Bosque Montano Alto» forma el orden de Purdiaeaetalia nutantis y crece sobre Histic Petraquepts. Representando una vegetación monotípica con un solo estrato arbóreo de 5-10 m $(-15 \mathrm{~m})$ de altura, completamente dominado por Purdiaea nutans (Cyrillaceae). El bosque ubicado a mayor altitud en el área, densamente enlazado con el páramo es el «Bosque Nublado Subalpino». Descritos como Clusio ellipticae-Weinmannietum cochensis aparecen más como una vegetación arbustiva casi impenetrable, y crecen sobre Humaquic Epiaquents. El límite del bosque es causado principalmente por los vientos fuertes de la región. Derrumbes e incendios naturales representan el «motor» de regeneración de los bosques. Se encontró carbón en la base de los niveles $\mathrm{A}$ de muchos perfiles de suelo. El análisis ${ }^{14} \mathrm{C}$ precisó una edad de 710-980 \pm 50 años antes del presente. Esto indica que fuegos en la vegetación han contribuido a la dinámica de esta vegetación en el pasado y no es un fenómeno reciente. El diámetro basal de los árboles y el volumen total de madera por hectárea disminuyen rápidamente con la altitud a partir de los $2100 \mathrm{~m}$ y no pueden ser eficazmente utilizados para una explotación de madera sostenible.

Palabras clave: Braun-Blanquet, fitosociología, bosque montano tropical, Andes, gradiente altitudinal, Ecuador.

\begin{abstract}
Few floristic inventories and even less syntaxonomical vegetation descriptions of tropical mountain forests exist. The author presents a syntaxonomical treatment of the vegetation of Reserva Biológica of San Francisco at the northern limit of Podocarpus National Park, Ecuador, together with notes on the corresponding soil types. The Lower Montane Forest (1800-2150 m), grouped in the new order Alzateetalia verticillatae, has a very diverse tree layer 20-35 m tall, and are a typical mosaic-climax. It grows on Terric Haplosaprists and Aquic Dystrupepts, developed from old landslide material and extends up to elevations of $2300 \mathrm{~m}$ at the bottom of wind-protected riverine valleys. At altitudes from 2100-2650 m (-2750 m), the forest structure and floristic composition change completely. The vegetation types belonging to this Upper Montane Forest form the newly described Purdiaeaetalia nutantis, growing on Histic Petraquepts. They represent a monotypic vegetation, with only one tree stratum, and stems between $5-10 \mathrm{~m}$, rarely up to $15 \mathrm{~m}$ tall. The canopy is completely dominated by the twisted stems of Purdiaea nutans (Cyrillaceae). The Subalpine-elfin forest which closely resembles that of the the Bolivian Jalca forms the uppermost forest belt of the study area. Described as Clusio ellipticae-Weinmannietum cochensis, this forest - more like an impenetrable bushland- grows on Humaqueptic Epiaquents and is closely dovetailed with the adjacent páramo region. The timberline in the area is mainly induced by strong winds. Charcoal was found at the base of the A horizons of many soil profiles up to the top region and $14 \mathrm{C}$ dated to $710-980+/-50$ years BP. This indicates that vegetation fires occurred in the past, and are not only a recent phenomenon.
\end{abstract}

Key words: Braun-Blanquet, phytosociology, Tropical Mountain Forests, Andes, altitudinal gradient, Ecuador.

* Harold L. Lyon Arboretum, Universidad de Hawaii, 3860 Manoa Road, Honolulu, HI 96822, EE.UU.

E-mail: bussmann@hawaii.edu 


\section{Introducción}

Los bosques montanos tropicales representan uno de los ecosistemas más diversos del mundo. Especialmente los Andes orientales son uno de los «puntos calientes» de biodiversidad (Myers et al., 2000). Comparado con los bosques húmedos bajos, los bosques andinos han recibido poco interés de los científicos y del público en el pasado; a pesar de su función ecológica y económica sumamente importante por ejemplo, en la captación de agua y el control de la erosión. Al mismo tiempo, los bosques montanos representan un ecosistema muy frágil por sus fuertes pendientes que los hacen vulnerables a una erosión extremadamente acelerada en condiciones de intensas lluvias. El incremento de la población y el incremento de la necesidad por recursos (leña, recursos minerales, pastizales, agricultura) ha venido disminuyendo la extensión del bosque montano continuamente. La mayoría de los estudios en ecosistemas tropicales tuvieron su enfoque en los bosques húmedos tropicales, donde se encuentra también la mayoría de las estaciones científicas (Leigh, 1999). No obstante, se sabe muy poco sobre los procesos de regeneración en estos ecosistemas (Finegan, 1996), y casi nada sobre su funcionamiento. En los ecosistemas de la montaña tropical el desconocimiento es mayor, aquí los estudios se concentraron en la zona andina, mientras la zona del bosque montano muchas veces con acceso muy difícil y una inmensa diversidad de especies casi no ha sido estudiado (Gentry, 1995; Webster, 1995). Aunque la biodiversidad del bosque andino es verdaderamente increíble (Barthlott et al., 1996; Ibisch, 1996), frecuentemente comparable e inclusive más alta que en áreas similares de la Amazonía (Balslev et al., 1998), casi no existen tratamientos fitosociológicos amplios de bosques montanos neotropicales. Meier (1998) presenta el único estudio con enfoque de la vegetación para el Parque Nacional Ávila en Venezuela.

Incluso proyectos grandes como Ecoandes en Colombia (Hammen et al., 1983, 1984,
1989, 1995) incluyeron la región de los bosques sólo de una manera marginal. Aunque la mayoría de la flora vascular en bosques montanos pertenece a formas de vidas no-leñosas (Gentry \& Dodson, 1987a, 1987b; Ibisch, 1996; Balslev et al., 1998; Galeano et al., 1999), la mayor parte de los estudios tiene sólo el enfoque de especies arbóreas (Gentry, 1988; 1995; Kitayama, 1992; Aiba y Kitayama, 1999). Con frecuencia las investigaciones se limitaron a pocas áreas y niveles de altitud, existiendo escasos estudios de gradientes altitudinales más completos. Las pocas publicaciones sobre la vegetación de los bosques montanos de Ecuador sólo contienen listas de especies o mencionan la región montañosa en comparación de los bosques de la Amazonía (Grubb et al., 1963; 1966; Ek, 1997). Los primeros esfuerzos para unir este conocimiento inicial se efectuaron recientemente (Hamilton et al., 1994; Churchill et al., 1995). El déficit de información científica incluye, especialmente, los aspectos de regeneración y uso de los bosques montanos y los procesos de sucesión después de impactos naturales o antrópicos. Los requisitos del hábitat y el potencial para regeneración de las especies maderables importantes son casi completamente desconocidos.

El objetivo del presente trabajo es documentar la vegetación y su zonación para los bosques montanos y la región subalpina en el sur de Ecuador.

\section{Área de estudio}

La región fronteriza de Ecuador y Perú pertenece a las áreas biológicamente más diversas del mundo y por esto es un «punto caliente» de biodiversidad por excelencia. Pasos bajos en la cadena andina permiten el intercambio fácil entre las floras y faunas de la Amazonía y la zona baja del Pacífico. Adicionalmente la región muestra una transición muy rápida entre los Andes húmedos del norte y los bosques secos deciduos de la zona baja del norte de Perú. Hasta hace poco, el Parque Nacional Podocarpus y el área del presente estudio casi eran científicamente 


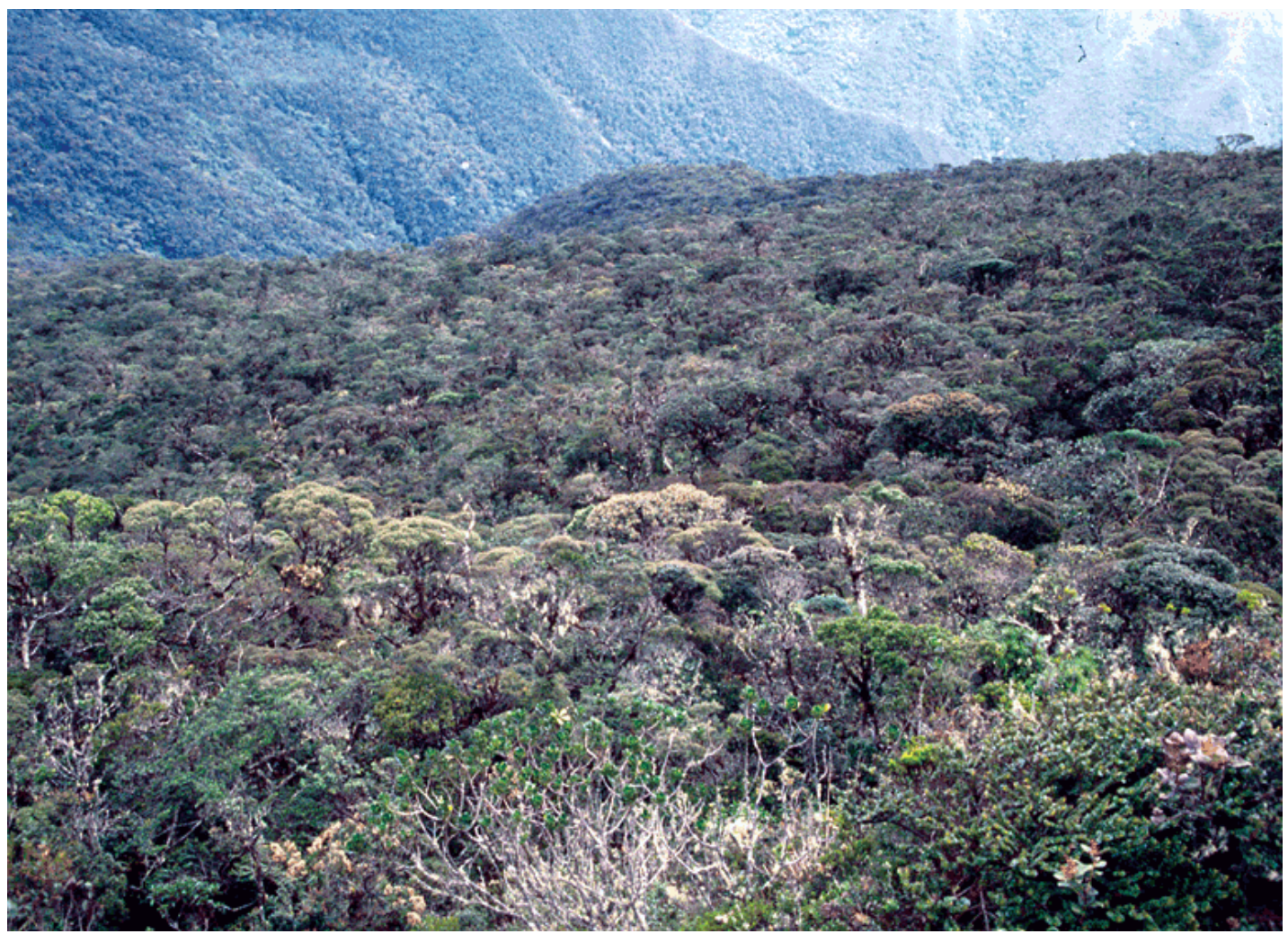

Figura 1. Vista panorámica del bosque de la Reserva Biológica San Francisco.

desconocidos. Existen pocos estudios sobre la flora de la Provincia de Loja (Espinosa 1948a,b; Emperaire y Friedberg, 1990; Ollgaard y Madsen, 1993; Ulloa y Joergensen, 1993; Joergensen y Ulloa, 1994; Madsen y Ollgaard, 1994; Bussmann y Lange, 1998; Joergensen y León-Yanez, 1999; Bussmann, 2001), o brindan descripciones breves de su vegetación (Espinosa, 1989, 1992; Madsen, 1989, 1991; Joergensen, 1991; Bogh, 1992; Keating, 1995, 1997, 1998, 1999, 2000). El esfuerzo más reciente para la clasificación de la vegetación de Ecuador (Sierra, 1999), refiere a todos los bosques montanos entre 1800-3000 m de altitud como «bosque de neblina montano» $\sin$ mayor distinción.

Estudios sobre la composición y regeneración de los bosques de la Reserva Biológica San Francisco se elaboraron desde 1997 en el marco del proyecto «Funcionalidad en un bosque montano tropical: Diversidad, procesos dinámicos y potencial para el uso» de la Fundación Alemana para la Investigación (DFG). La intención de las investigaciones fue de caracterizar los diferentes tipos de bosques y sus estados de regeneración a lo largo de la gradiente altitudinal, además de evaluar si se puede elaborar una caracterización detallada sólo sobre la base de un estudio fisonómico o si se necesita un análisis fitosociológico detallado.

La reserva biológica San Francisco se encuentra entre las capitales provinciales de Loja y Zamora e incluye 1000 hectáreas de la extensión norte de la cordillera de Consuelo, a $03^{\circ} 58^{\prime} 18^{\prime \prime} \mathrm{S}-79^{\circ} 04^{\prime} 44^{\prime}$ ' O, en la provincia Zamora-Chinchipe, Ecuador, bordeado por el parque nacional Podocarpus, la única área protegida en el sur del país. La reserva biológica San Francisco se distribuye entre los 1800$3150 \mathrm{~m}$ y forma un transecto completo de los bosques montanos de la región y áreas antrópicamente perturbadas que pueden ser comparados (Fig. 1). La topografía es inmensamente escarpada con pendientes entre $40-60^{\circ}$, 1legando con frecuencia a $90^{\circ}$. Los bosques son accesibles por cuatro senderos. 


\section{Geología y suelos}

En la parte sur de Ecuador y el norte de Perú la mayor elevación es de 4600 m, y en gran parte no supera los $4000 \mathrm{~m}$ (Joergensen y Ulloa, 1994), siendo los Andes ecuatoriales el área que representa la región más baja. Mientras la roca base de los Andes del norte está formada por vulcanismo Cuaternario, la parte sur se encuentra formada por sedimentos Terciarios (Hall, 1977). Los sustratos geológicos consisten en roca arenosa y piedra azul. La mayoría de los suelos tiene una capa orgánica muy amplia de hasta $48 \mathrm{~cm}$ de espesor. Los suelos de la parte baja (hasta 2100 m) consisten de Dystrudepts y Haplosaprists, desarrollados sobre material de derrumbes, rico en piedras. Carbón encontrado a esta altitud tuvo una edad de $710+/-50$ años antes del presente, según la datación ${ }^{14} \mathrm{C}$, sugiriendo que los derrumbes ocurrieron después de la quema de los bosques originarios. Estos suelos todavía contienen un alto porcentaje de roca en proceso de descomposición. En alturas mayores (2100-2700 m) el tipo común de suelos es el Petraquept, que por lo menos en parte se ha desarrollado sobre material de soliflucción, seguido por Epiaquepts en la zona más alta. Se encontró carbón debajo del estrato A de muchos perfiles de suelos hasta la zona más alta, con datación ${ }^{14} \mathrm{C}$ de $710-980+/-50$ años antes del presente. Esto indica que las quemas tuvieron un proceso continuo y que no se trata de un fenómeno reciente (Schrumpf et al., 2001).

\section{Condiciones climáticas}

Emck (en prep.) describe una precipitación anual promedio de $2500 \mathrm{~mm}$ en la zona baja y más de $5000 \mathrm{~mm}$ en las zonas más altas de la reserva, con temperaturas anuales promedio entre $15-17{ }^{\circ} \mathrm{C}$ y $9-11^{\circ} \mathrm{C}$ en las mismas regiones, registradas durante 1997-2001. Especialmente la parte más alta se encuentra con nubes o neblina casi todo el año. Las precipitaciones en febrero-marzo consisten en lluvias fuertes casi diarias muy regulares, mientras las de junio-septiembre son lluvias continuas finas con vientos fuertes. No se encuentra una diferencia mayor en el monto acumulado entre estos meses. Entre octubre-enero las 1luvias consisten en precipitaciones muy fuertes pero irregulares, llegando casi al monto de los otros meses. No obstante, los intervalos de sol entre las lluvias fuertes causan condiciones para un balance de agua negativo por unos días y de hasta 3-4 semanas durante estos meses secos, aunque el diagrama climatológico no muestra períodos áridos. Esto explica las adaptaciones xeromórficas de muchas especies del bosque aunque éste se halle cubierto por nubes la mayoría del tiempo.

\section{Material y métodos}

El trabajo de campo en la reserva se inició en septiembre de 1995 y viene siendo continuado, permitiendo el control de parcelas de estudio durante todas las temporadas del año, incluyendo un evento fuerte del ciclo El Niño/La Niña en 1997-1998. Después de un estudio florístico detallado, los bosques de la reserva biológica San Francisco (ECSF) han sido investigados en sus características fisionómicos y fitosociológicos. Un total de 307 parcelas nopermanentes en áreas de bosques homogéneos, 76 parcelas en derrumbes naturales y 40 en derrumbes antropogénicos fueron investigadas con la metodología de Braun-Blanquet (1964) descrita por Mueller-Dombois y Ellenberg (1974) y modificada por Hammen et al. (1989). El tamaño de las parcelas (mínimo $400 \mathrm{~m}^{2}$ en bosques y $4 \mathrm{~m}^{2}$ en caso de derrumbes) siempre fue mayor que el área mínima determinada (2 $\mathrm{m}^{2}$ para derrumbes, $256 \mathrm{~m}^{2}$ para el bosque montano bajo y $64 \mathrm{~m}^{2}$ para el bosque montano alto y áreas de Jalca), con el objetivo de obtener datos más exactos sobre cobertura y abundancia, pero suficientemente pequeño para mantener los factores abióticos homogéneos en la parcela. En general, las parcelas tuvieron una forma rectangular, pero especialmente en hábitats lineares como bordes, derrumbes, etc., se seleccionaron formas diferentes.

Se identificaron tres estratos de vegetación: $\mathrm{T}=$ Estrato de árboles (plantas leñosas $>5 \mathrm{~m}$ ), 
$\mathrm{S}=$ Estrato arbustivo (plantas leñosas 0,50-5 $\mathrm{m}$ ), $\mathrm{H}=$ Estrato herbáceo (plantas herbáceas $<0,50 \mathrm{~m})$.

Para permitir una mejor comparación con datos de otras regiones se instalaron 14 parcelas de 0,1 hectárea siguiendo 2 transectos, para investigar la estructura de la vegetación arbórea. Cada $100 \mathrm{~m}$ de altitud se instalaron dos de estas parcelas de $20 \times 50 \mathrm{~m}$. Se registraron altura y diámetro a la altura del pecho (dap) de todos los árboles con un dap $>10 \mathrm{~cm}$, marcando todos los individuos con placas de aluminio $\mathrm{y}$ anotando todas las especies presentes.

Las muestras colectadas fueron secadas en el herbario de la estación científica San Francisco (ECSF), e identificadas en los herbarios ECSF, Loja y el Herbario Nacional de Ecuador (QCNE), y registrado en la base de datos de Loja. Un juego completo de las colecciones se encuentra en cualquiera de estos herbarios. La nomenclatura de los especies sigue el catálogo de Joergensen y LeónYañez (1999), la de las unidades de vegetación sigue a Barkmann et al. (1986).

Se registraron presencia y ausencia de todas las especies en cada parcela. Especies epífitas se recolectaron de árboles y ramas caídas, y escalando árboles selectos, con podadoras de $8 \mathrm{~m}$, y por observación con binoculares (incluso fuera de las parcelas para tener un inventario completo de la flora epífita). En nueve parcelas y a diferentes altitudes se registró y mapeó la flora epífita entera de árboles seleccionados y se anotó la distribución en las zonas de Johansson (Johansson, 1974). Hasta ahora se han registrado 2258 especies de plantas vasculares en 636 géneros de 176 familias en la reserva biológica San Francisco.

\section{Resultados}

\section{Sinopsis de las comunidades vegetales de la reserva biológica San Francisco}

Se puede agrupar la vegetación de reserva biológica San Francisco en cuatro formaciones forestales grandes, distinguiendo las zonas altitudinales más obvias. Pendientes muy escarpadas, quebradas muy profundas, produciendo una variedad de microclimas y la frecuencia de derrumbes naturales causan un mosaico de diferentes tipos de vegetación en el sur de Ecuador (Bussmann, 2001): E1 Bosque Montano Bajo (1800-2150 m), Bosque Montano Alto (2150-2650 m), Jalca (Bosque Subalpino, Ceja Andina, 2650-3000 m) y Páramo (2700-3150 m). Especialmente las comunidades de Jalca y Páramo se encuentran muy entrelazadas cerca del límite del bosque.

Bosque Montano Bajo - Alzateetalia verticillatae. Esta orden incluye las unidades de bosque entre $1800-2150 \mathrm{~m}$, con un estrato arbóreo muy diverso, 20-35 m de alto, con 2-3 estratos, representando un clímax mosaico muy bien desarrollado de numerosas especies, con pocas especies de gran cobertura/abundancia. Crece sobre Terric Haplosaprists y Aquic Dystrupepts (Schrumpf et al., 2001). Este Bosque Montano Bajo (bosque de Ocotea y Nectandra; Bussmann, 2001), se extiende hasta un nivel de $2300 \mathrm{~m}$ en quebradas protegidas del viento fuerte de las zonas altas. Especies de familias de la zona húmeda tropical, normalmente encontradas en altitudes más bajas (e.g. Cyclanthaceae, Lauraceae y Hymenophyllaceae) se hallan con frecuencia, mientras representantes de la flora de la zona más alta tienen poca presencia. Áreas no-perturbadas de los Alzateetalia se encuentran casi sólo en pendientes de 30-50\%, o más, y en valles casi inaccesibles. En áreas de acceso fácil las Alzateetalia han sido destruidas casi por completo por actividades antrópicas, y fueron reemplazadas por bosques secundarios. Con casi $2500 \mathrm{~mm}$ de precipitación anual las Alzateetalia verticillatae representan los tipos de bosques más secos del área de estudio, creciendo especialmente sobre Typic Tropothents y Oxaquic Humitropepts (Schrumpf et al., 2001).

Con 880-1210 árboles de más de $10 \mathrm{~cm}$ diámetro a la altura del pecho (dap)/ha, el Bosque Montano Alto muestra la mayor abundancia de árboles en el área de estudio, y una de las presencias más numerosas encontra- 
das en bosques montanos hasta ahora. Con 339 especies, las epífitas son la forma de vida más diversa en estos bosques, seguida por los árboles (318 especies), hierbas (186), arbustos (134), helechos no-epífititos (108), trepadoras (26) y parásitos (4). La mitad de los géneros de Araceae encontrados en ECSF crecieron sólo en esta zona baja. Orquídeas son el grupo de epífitas dominante (153 especies), con casi la mitad de los géneros restringidos a esta zona.

Bosque Montano Alto - Purdiaeaetalia nutantis. La posición sintaxonómica del Bosque Montano Alto todavía requiere clarificaciones. Este orden nuevo muestra relaciones al grupo de comunidades de Hedyosmum pseudoandromeda establecido por Meier (1998). En altitudes desde 2100-2650 (<2750 $\mathrm{m})$, la estructura y composición florística del bosque cambian por completo, con los fustes torcidos, pequeños y cubiertos por líquenes. Este Bosque Montano Alto (Bosque de Purdiaea nutans, Myrica pubescens, Myrsine andina; Bussmann; 2001) reemplaza los Alzateetalia verticillatae. Representa un tipo de vegetación monotípica, con un solo estrato arbóreo 5-10 m, raramente $15 \mathrm{~m}$ de altura, creciendo sobre Histic Petraquepts (Schrumpf et al., 2001). Especies de la zona baja desaparecen completamente. Los fustes torcidos de Purdiaea nutans (Cyrillaceae), la cual tiene su distribución mayor en el norte de Perú, dominan el dosel. En muy pocos lugares se pueden encontrar especies diferentes con abundancia co-dominante. Se observa, asimismo, un estrato muy diverso de arbustos y arbolitos. Muchas especies de los Purdiaeaetalia tienen hojas xeromórficas como adaptación a la radiación solar muy alta y como protección durante los meses secos. El Bosque Montano Alto se encuentra especialmente sobre Oxaquic y Aquic Dystropepts, con una precipitación anual de casi $4000 \mathrm{~mm}$. El número de árboles diminuye claramente con la altura: a $2225 \mathrm{~m}$ aún se encuentran 650 árboles/ha, disminuyendo a 160 árboles/ha a los $2425 \mathrm{~m}$, donde el bosque parece ya muy «abier- to». Las epífitas siguen siendo la forma de vida más abundante con 283 especies. La diversidad de árboles y pteridofitos no-epífititos disminuye rápidamente (a 192 y 63 especies respectivamente), mientras las hierbas (159) y arbustos (131) se vuelven más importantes que en el Bosque Montano Bajo, como también trepadoras (24) y parásitos (13). Aunque la mayoría de las familias importantes siguen presentes, su número de especies decrece, con la notable excepción de las orquídeas. Especialmente Pleurothallidinae (Lepanthes, 32 especies; Pleurothallis 25 especies) tienen su centro de diversidad en estos bosques de neblina. Polypodiaceae epífititas pequeñas (así como Terpsichore y Melpomene) y también Hymenophyllaceae se encuentran con el mayor número de especies en esta altitud.

Jalca (Bosque Subalpino, Ceja Andina) - Clusio ellipticae - Weinmannietalia cochensis. Bosque Subalpino (Bussmann, 2001) es la vegetación boscosa en las zonas más altas de la ECSF y se parecen a la Jalca de Bolivia. Esta formación es más un estrato arbustivo impenetrable que un verdadero bosque y crece sobre Humaqueptic Epiaquents (Schrumpf et al., 2001); se encuentra asimismo muy entrelazado con las áreas de páramo. El límite de bosque en el área está principalmente inducido por los vientos fuertes. Áreas protegidas del viento se hallan densamente cubiertas por vegetación de Jalca, mientras áreas abiertas, expuestas a los vientos fuertes en la misma altitud muestran la vegetación del páramo. Un verdadero límite de bosque no existe en el área de estudio por esta razón. Desde una altitud de $2450 \mathrm{~m}$ hacia arriba se encuentran islas de Jalca en el Bosque Montano Alto. Las copas de sólo 1-2 m de ancho de las especies dominantes, especialmente Cunoniaceae (Weinmannia spp.), Clusiaceae (Clusia spp.), Clethraceae (Clethra spp.) y muchas especies pequeñas de Melastomataceae (Brachyotum spp., Miconia spp.), forman un dosel muy denso, permitiendo sólo muy poca luz al llegar al suelo. Los fustes de estos árboles casi nunca lle- 


\section{Regeneración}

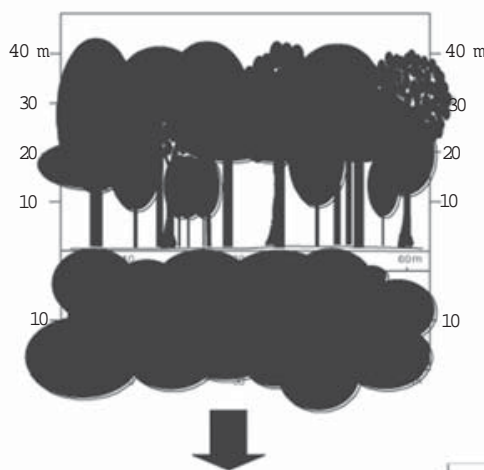

Muerte de árboles del bosque primario permite regeneración de especies secundarias. Establecimiento de especies primarias bajo la sombra de las pioneras
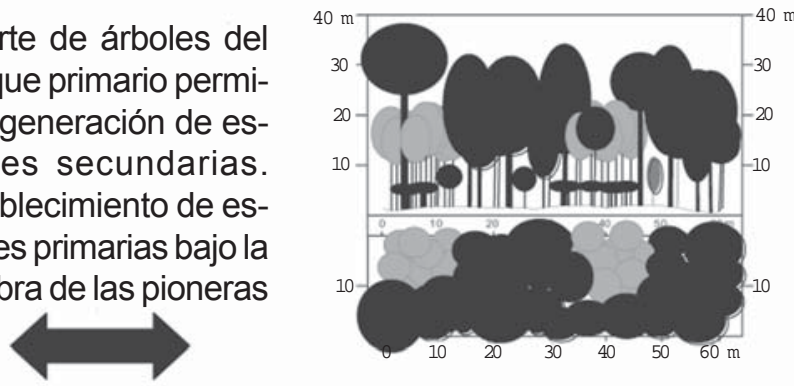

Tala intensiva causa cambio de estructura del bosque por un bosque secundario permanente, con ciclo de regeneración diferente.
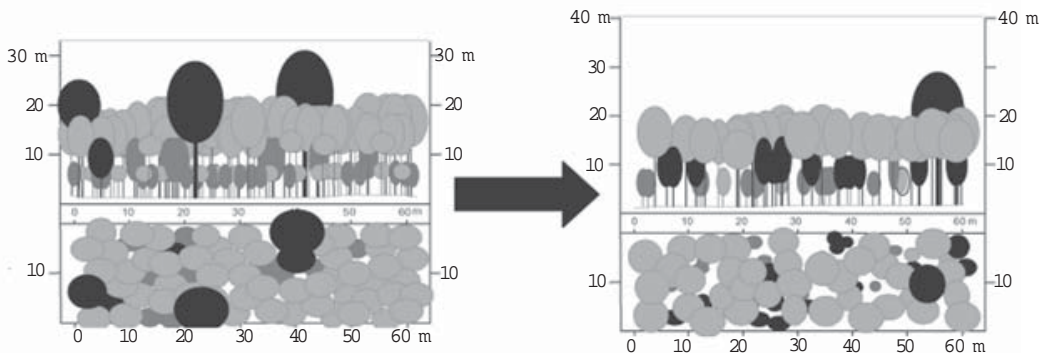

Figura 2. Regeneración en el bosque montano bajo.

gan a un dap mayor que $10 \mathrm{~cm}$, salen de una alfombra de briofitos muy poco profunda. Los árboles representan la forma de vida más abundante con 172 especies, arbustos (131), hierbas (132), helechos terrestres (37), trepadoras (15) y parásitos (2). Sólo se registraron 120 especies de epífitas en la Jalca. Familias de la zona baja como Araceae y Piperaceae desaparecieron, la diversidad de bromelíaceas y orquídeas disminuyó. Mientras en otros tipos de bosque las orquídeas representaron más o menos una tercera parte de las epífitas, en la Jalca su importancia llega a 60\%. Este nivel es parecido a lo documentado por Bogh (1992): 138 especies en una parcela en el área cercana de Cajanuma. Las comunidades de Jalca crecen sobre Oxaquic y Aquic Dystropepts y reciben una precipitación anual de casi $4000 \mathrm{~mm}$. La ocurrencia casi constante de neblina procura humedad adicional.

\section{Regeneración}

En el Bosque Montano Bajo del sur de Ecuador los claros están colonizados de inmediato por Cecropiaceae (especialmente Cecropia montana), Piptocoma discolor (Asteraceae), Isertia laevis (Rubiaceae) y
Heliocarpus americanus (Tiliaceae). El dosel se cierra muy rápidamente y las especies primarias se regeneran bajo la sombra de las pioneras (Fig. 2).

La dinámica de los bosques sobre derrumbes fue estudiada por Stern (1995) en los Andes Ecuatorianos, por Kessler (1999) en Bolivia y por Ericksen et al. (1989) en los Andes centro-sur. Otros trabajos sobre derrumbes en el ambiente tropical incluyen Garwood et al. (1979) en Panamá, Guariguata (1990) en Puerto Rico y Bartarya y Valdiya (1989) en el Himalaya (India). Keefer (1984) estudió derrumbes a un nivel global. En estas regiones tropicales los derrumbes son muy frecuentes. En adición a pendientes escarpadas y lluvias fuertes, la construcción de carreteras, tala y quema debilitan el substrato, y la descomposición del material geológico también tiene una influencia en la frecuencia de derrumbes (Brabb y Harrold, 1989).

La función de los derrumbes para el desarrollo de la vegetación de esta región es muy importante, pues ellos representan un factor sumamente importante para el mantenimiento de la biodiversidad, la dinámica y la estabilidad del 
bosque montano. En bosques muy viejos, especialmente en el Bosque Montano Alto, la diversidad está drásticamente reducida. Mientras al otro lado la mayoría de las especies encontradas durante el proceso de la sucesión no constituyen elementos del bosque maduro. Por eso, los derrumbes son un factor muy importante para la regeneración del ecosistema.

La sucesión de derrumbes en Ecuador sigue más o menos el esquema encontrado en otras áreas de montaña (Herzog, 1923; Simonett, 1967; Garwood et al., 1979; Guariguata, 1990; Stern, 1995). No obstante, como Kessler (1999) ya observó en Bolivia, la regeneración de derrumbes andinos muestra diferencias en relación con la función de diferentes helechos. Como en Bolivia, Pteridium arachnoideum - aunque muy importante en la sucesión de áreas antrópicamente perturbadas-, no tuvo ninguna importancia en la regeneración de derrumbes naturales en el sur de Ecuador, con Gleicheniaceae encontrados como los helechos de mayor importancia. También, a diferencia a Stern (1995) y Kessler (1999), el bambú
(Chusquea spp.) no tuvo ninguna importancia en la regeneración de derrumbes en la estación científica San Francisco ni tampoco otras gramíneas como Cortaderia. Al contrario de las observaciones de Kessler (1999), casi no se observaron bosques muy viejos dominados por helechos o bambú. Gramíneas como Neurolepis elata o Rhynchospora locuples se encontraron principalmente en bosques alterados (por incendios) o muy antiguos (degenerados). Estas sinopsis indican que los procesos de sucesión en bosque montano siguen un solo patrón. Estudios adicionales a lo largo de los Andes deben de agregar muchos datos adicionales para un mejor entendimiento de los procesos de regeneración y sucesión a largo plazo.

En situaciones naturales, la primera fase de sucesión de derrumbes está dominada por briofitos, especialmente Polytrichum, y un estrato diverso de líquenes. Después de un tiempo entran especies de plantas vasculares. La duración de la regeneración depende mucho de la intensidad de actividad en el derrumbe. En promedio, la fase de criptógamas dura más o menos cinco años. En localidades con suelo muy

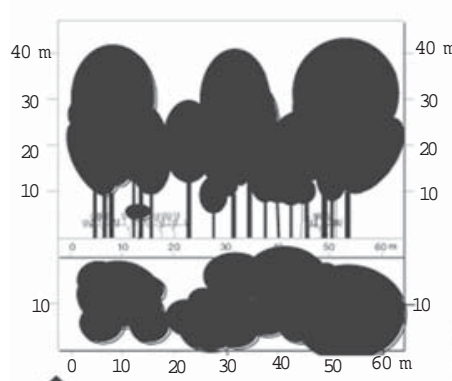

Periodos extensos sin perturbación

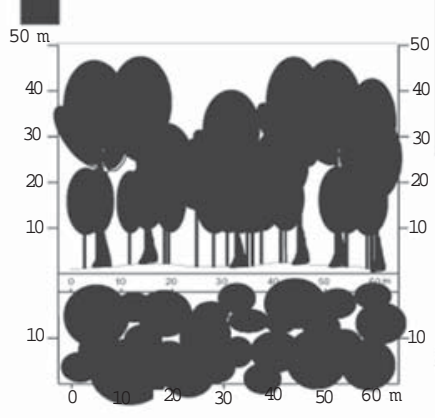

\section{Regeneración}

Después de un fuego o derrumbe natural, regeneración rápida del bosque primario. Formación de bosque monotípico. En ausencia de perturbación cambio de la estructura y composición florística del bosque.

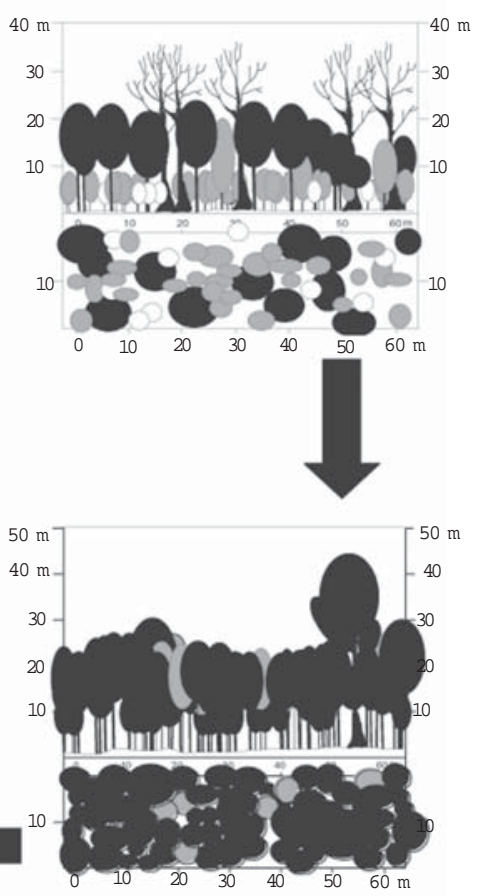

Figura 3. Regeneración en el bosque montano alto. 
rocoso la erosión puede continuar por mucho tiempo. En esta situación, representada con frecuencia al lado de carreteras, la fase primaria se puede extender por mucho tiempo.

La segunda fase de sucesión muestra un incremento de la densidad vegetal. Individuos de Gleicheniaceae y Lycopodiaceae colonizan los derrumbes. Lycopodiella glaucescens y Lycopodium clavatum crecen más rápido que las Gleicheniaceas, pero forman colonias menos densas. Esta fase secundaria está principalmente caracterizada por competencia con los pioneros de la primera fase, y por parte ya empieza la regeneración de especies arbustivas como Tibouchina lepidota, Graffenrieda harlingii (Melastomataceae) o Bejaria aestuans (Ericaceae). Estas pioneras no obstante no se encuentran en la comunidad clímax. En una fase tercera, la vegetación está dominada por arbustos como Ageratina dendroides (Asteraceae), y al fin se encuentran especies del bosque secundario.

En áreas demasiada viejas del Bosque Montano Alto y Jalca en el sur de Ecuador, el piso del bosque se encuentra densamente cubierto por Neurolepis elata (Bambusoideae), y el dosel parece muy abierto y disminuido, con una cubierta de menos del 5\%. Presenta un monto elevado de material orgánico seco, especialmente en eventos de floración masiva de Neurolepis y de la misma manera durante períodos de sequía (e.g. durante períodos de E1 Niño/La Niña), siendo muy susceptibles a incendios. Después de la quema, áreas abiertas son colonizadas rápidamente por especies de helechos oportunistas, pero sin ningún estadio de musgos y líquenes. En un tiempo muy corto se establecen especies arbóreas, en particular Purdiaea nutans, llegando de los bosques primarios de los alrededores, y después de un par de años las bromeliáceas características hacen su arribo. Debido al crecimiento muy lento de las especies dominantes, se desarrolla un estrato arbustivo muy denso, que se puede mantener por muchos años (Figs. 3, 4). Purdiaea nutans empieza a sobrepasar el resto de la vegetación lentamente, formando el típico clímax monotípico. Después de más o menos 400-500 años, este es-

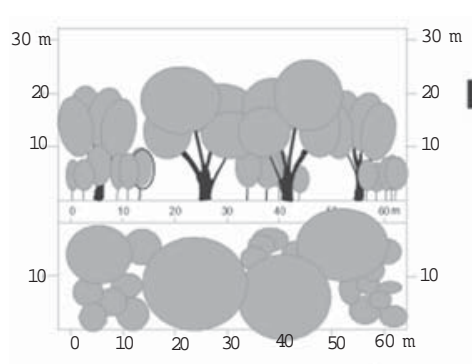

\section{Regeneración}

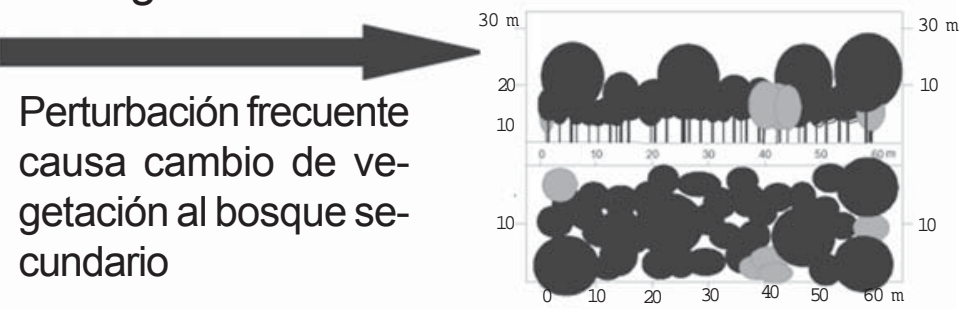

Comunidad clímax sin regeneración de especies de árboles dominantes. Después de un impacto (fuego) se observa rápida germinación de especies del bosque primario.

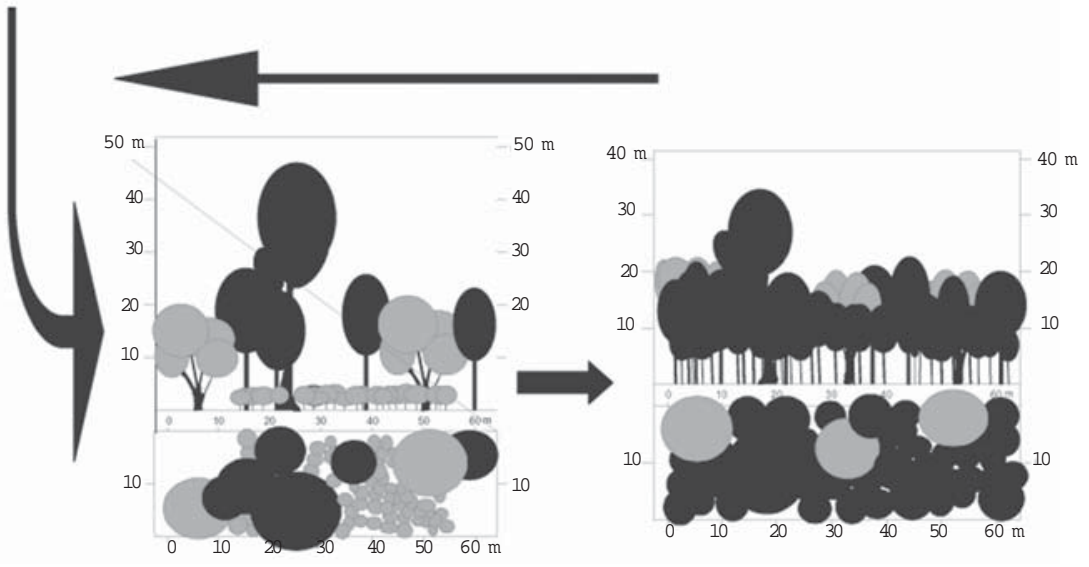

Figura 4. Regeneración en la Jalca. 
trato denso de Purdiaea empieza a morir, y el estrato denso de bromeliáceas y arbustos se cambia por las bambusoideas mencionadas, que toleran mucha insolación.

\section{Uso y manejo del bosque}

El sector forestal y la industria maderera de las provincias de Loja y Zamora-Chinchipe están viviendo una crisis acelerada y profundizada por la mala situación económica del país. Se puede observar la destrucción de los bosques para establecer pastizales, así como también la tala selectiva y la ausencia de reforestación con especies nativas. La escasez de madera es notoria y se suma a la mayor demanda de especies indígenas, mientras que especies exóticas como Pinus radiata, $P$. patula y Eucalyptus globulus no reciben el interés del consumidor. De las especies maderables usadas en el ámbito local, especialmente el cedro(Cedrela montana), el seique (Cedrellinga cateniformis), el almendro (Swietenia macrophylla), el nogal (Juglans neotropica), yumbingue (Terminalia amazonica) y el romerillo fino (Podocarpus oleifolius) son las más difíciles de encontrar, y además son taladas sin consideración de su edad o calidad. El Plywood, MDF y el «metal» están siendo usados cada vez más para combatir la escasez de material.

La disminución de ciertos tipos de madera en el mercado se asocia mayormente a las variaciones climáticas, restricciones del Ministerio del Medio Ambiente, mayor competencia y constituyen el factor más importante de la «falta de materia prima». Para evitar la pérdida de los bosques naturales el Ministerio de Medio Ambiente puso restricciones para garantizar la cosecha controlada. Cada propuesta de tala requiere un permiso escrito del ministerio para cortar y de la misma manera se controla el transporte de la madera. Sin embargo, estas medidas de control no son conocidas por la población rural, y la tala ilegal se observa con mucha frecuencia. El control policial incrementa los precios de la madera y su consiguiente escasez. Más o menos $50 \%$ de la madera en el mercado viene de la tala ilegal.
Más del $80 \%$ de la madera usada en Loja proviene de los bosques naturales de Zamora. Sólo pocas especies son taladas en los bosques del sur de la provincia de Loja. La mayoría de los productos de madera producidos en Loja o Zamora son vendidos dentro de las provincias. No obstante, una parte considerable se vende en las provincias aledañas y al Perú. Zamora sólo tiene un papel muy pequeño en el mercado interprovincial. La mayoría de los productos permanece en la ciudad o se vende a Loja.

El fenómeno climático «El Niño» tiene cierta influencia en las oscilaciones del mercado de madera, porque durante temporadas con lluvias fuertes la única carretera entre Loja y Zamora se encuentra bloqueada con frecuencia. Este problema no obstante se mencionó sólo en Loja, y no tiene influencia por la disponibilidad de madera en Zamora. Puede ser que la migración de una gran parte de mano de obra cause una escasez fuerte de mano de obra, y de esta manera una disminución en la producción.

En adición a las actividades frecuentes de tala ilegal, el procesamiento muy ineficaz de la madera y las prácticas no-sustentables de tala, son los factores más importantes para la destrucción de los bosques de Ecuador. La mayoría de los árboles talados son cortados en tablas directamente en el bosque, usando motosierras. Esto causa una pérdida en forma de polvo de madera de hasta $50 \%$ de la materia prima. La maquinaria muy vieja de la mayoría de aserraderos y carpinterías — por falta de recursos económicos - incrementa la pérdida del material que, hasta el producto final, puede alcanzar $75 \%$.

\section{Discusión}

Se puede agrupar los tipos de bosque montano mayores encontrados en la reserva biológica de San Francisco en tres formaciones. La riqueza de la flora en estos bosques montanos está dada por las hierbas, arbustos y epífitas (Gentry, 1988, 1995; Gentry y Dodson, 1987), mientras que los árboles son menos diversos en comparación con los bos- 
ques húmedos tropicales, una metodología con enfoque no sólo en las grandes especies arbóreas permite un acceso mucho más detallado de esta diversidad. Los bosques montanos estudiados mostraron diferencias muy profundas con otras áreas en la zona de amortiguamiento, y también con la vegetación de otras áreas del Ecuador. La abundancia muy alta de Alzatea verticillata (Alzateaceae) una familia que antes no hubiera sido encontrado en Ecuador. El Bosque Montano Bajo es único, aunque la composición florística de esta formación forestal, en general, se puede comparar a otras áreas (Frei, 1958; Grubb et al., 1963; 1966; Madsen, 1989, 1991; Madsen \& Oellgaard, 1994; Meier, 1998). Al contrario, se debe interpretar el Bosque Montano Alto, dominado por Purdiaea nutans, como formación muy especial y aislada, con solamente pequeñas áreas comparables ubicadas en el norte de Perú. Es así como en el área cercana a Cajanuma, la composición de los bosques en esta altura es completamente diferente (Madsen, 1989, 1991; Madsen y Oellgaard, 1994). Florísticamente los bosques de la ECSF no son representativos para un área de bosque nublado del sur de Ecuador, y menos para el bosque andino. Sistemas de clasificación basados en criterios estructurales (Paulsch y Czimczik, 2001) proponen un conjunto muy similar de tipos de bosques.

Los derrumbes juegan un papel muy importante en el desarrollo de la vegetación de esta región. La mayoría de las especies encontradas durante el proceso de la sucesión no son elementos del bosque maduro. Por esto, los derrumbes son un factor muy importante en la regeneración del ecosistema. La sucesión de derrumbes sigue más o menos el esquema encontrado en otras áreas de montaña (Herzog, 1923; Simonett, 1967; Garwood et al., 1979; Garwood, 1985; Guariguata 1990; Stern, 1995). No obstante, como Kessler (1999) observa en Bolivia, la regeneración de derrumbes andinos muestra diferencias con relación a la función de diferentes helechos. Como en Bolivia, Pteridium arachnoideum -aunque muy importante en la sucesión de áreas antrópicamente perturbadas-, no tiene ninguna importancia en la regeneración de derrumbes naturales en el sur de Ecuador, con Gleicheniaceae encontrados como los helechos de mayor importancia. A diferencia de los propuesto por Stern (1995) en el norte de Ecuador y Kessler (1999), el bambú (Chusquea spp.) no tuvo ninguna importancia en la regeneración de derrumbes en ECSF. Las gramíneas como Cortaderia tampoco tuvieron una influencia larga en los proceso de sucesión. Contrariamente a las observaciones de Kessler (1999), casi no se evidenciaron bosques muy viejos dominados por helechos o bambú. Sin embargo, gramíneas como Neurolepis elata o Rhynchospora locuples se encontraron mayormente en partes donde la comunidad terminal probablemente fue destruida por fuegos naturales o estuvo muy vieja. Se deben iniciar investigaciones de los procesos de sucesión a largo tiempo en toda la región andina para salir con datos generales sobre la regeneración.

Si el uso actual de los bosques naturales continúa, las condiciones rápidamente se volverían críticas para la industria maderera. Para evitar la crisis se tienen que tomar medidas rápidas:

- Disminuir la pérdida de material durante la tala y procesamiento de la madera.

- Empezar con programas de reforestación con especies indígenas.

- Institucionalizar programas de educación sobre uso sostenible de los bosques.

Casi todas las organizaciones prefieren la segunda opción, debido a que la mayoría de las especies nativas tienen un crecimiento comparativamente lento; sin embargo, para esto se necesita una acción rápida para instalar sistemas agroforestales y silvipastorales. No obstante, con este escenario ideal la situación no mejorará en los próximos 10 años. Para mejorar el uso de la madera, carpinterías y aserradoras se necesita la posibilidad de recibir créditos baratos para mejorar la maquinaría; 
el establecimiento de un parque industrial en Loja sería el primer paso en esta dirección.

A largo plazo, los bosques naturales de la región son la fuente más importante de trabajo. Su protección no sólo depende de los agricultores y ganaderos, sino especialmente de los esfuerzos gubernamentales para difundir e implicar programas de manejo como el dar incentivos para la reforestación con especies nativas.

Como todavía los ciclos de reproducción, requisitos ecológicos y condiciones para una germinación óptima de la mayoría de especies nativas son desconocidos, más estudios aplicados al respecto deben de ser realizados para dar una base sólida a cualquier programa de reforestación.

\section{Agradecimientos}

Agradezco mucho el apoyo continuo de la Deutsche Forschungsgemeinschaft (DFG) en el programa «Funcionalidad de un bosque montano tropical: diversidad, procesos dinámicos y potencial para el uso». También quiero agradecer al Ministerio de Medio Ambiente / INEFAN por el permiso de investigación y a la Fundación Científica San Francisco. Un agradecimiento especial a mis colegas ecuatorianos y alemanes, y gracias también a mis estudiantes.

\section{Literatura citada}

Aiba, S. y K. Kitayama, 1999. «Structure, composition and species diversity in an altitude-substrate matrix of rain forest tree communties on Mount Kinabalu, Borneo». Plant Ecology, (149): 139157.

Balslev, H.; R. Valencia, G. Paz Y Miño, Christensen, H. y I. Nielsen. 1998. «Species count of vascular plants in one hectare of humid lowland forest in Amazonian Ecuador». En: F. Dallmeier y J.A. Comiseky (Eds.): Forest biodiversity in North, Central and South America, and the Caribbean, pp. 585-596. París: MAB.

Barkmann, J.J.; J. Moravec y S. Rauchert. 1986. Code of phytosociological nomenclature. Vegetatio, (67): 145-195.

Barthlott, W.; W. Lauer y A. Placke. 1996. Global distribution of species diversity in vascuar plants: towards a world map of phytodiversity. Erdkunde, (50): 317-327.
Bogh, A. 1992. Composition and distribution of the vascular epiphyte flora of an Ecuadorian montane rainforest. Selbyana, (13): 25-34.

Braun-Blanquet, J. 1964. Pflanzensoziologie (3. a edición). Nueva York: Springer, Wien,

Bussmann, R.W. 2001. The montane forests of Reserva Biologica San Francisco (Zamora-Chinchipe, Ecuador) - vegetation zonation and natural regeneration. Die ERDE, (132): 11-24.

Bussmann, R.W. y S. Lange 1998. Expedición Rápido Asesso Cordillera Sabanilla - Inventario florístico. Loja/Quito: INEFAN.

Churchill, S.P.; H. Balslev; E. Forero y J.L. Luteyn (Eds.). 1995. Biodiversity and Conservation of Neotropical Montane Forests. Nueva York: NYBG. 703 p.

Cleef, A.M. 1981. The Vegetation of the Páramos of the Colombian Cordillera Oriental. Dissertationes Botanicae: 61. Vaduz: Cramer.

Cleef, A.M.; Ch. O. Rangel.; T. Hammen, van der y M. R. Jaramillo. 1984. La vegetación de las selvas del transecto Buritica. En: Hammen, T. van der \& P.M. Ruiz (Eds.): Studies on tropical andean ecosystems vol. 2, pp. 267-406. Vaduz: Cramer.

Ek, R.C. 1997. Botanical diversity in the tropical rain forest of Guayana. Tropenbos-Guayana series, (4): 1-237.

Emck, P. en prep. Climatic conditions in the Cordillera de Numbala (Podocarpus National Park), Southern Ecuador. PhD tesis, University of Erlangen.

Emperaire, L. y C. Friedberg, 1990. Releves floristiques des regions Piura (Perou) et de Loja (Ecuateur). París: ORSTROM.

Espinosa, B. 1948a. Estudios botánicos en el sur del Ecuador. I. Loja-Catamayo-MalacatosVilcabamba. Loja: Universidad Nacional. - 1948b. Estudios botánicos en el sur del Ecuador. II. Herbarium Universitatis Loxoensis (Primer Inventario). Loja: Universidad Nacional.

Espinosa, G.D.A. (Ed.). 1989/1992. Parque Nacional Podocarpus. Boletín informativo sobre biología, conservación y vida silvestre, vol. 1-3. Loja: Universidad Nacional..

Finegan, B. 1996. Pattern and process in neotropical secondary rain forest: the first 100 ears of succession. TREE, (11): 119-124.

Frei, E. 1958. Eine Studie ueber den Zusammenhang zwischen Bodentyp, Klima und Vegetation in Ecuador. Plant and Soil, (9): 215-236.

Galeano, G.; S. Suárez y H. Balslev. 1999. Vascular plant species count in a wet forest in the Choco area on the Pacific coast of Colombia. Biodiv. Cons., (7): 1563-1575.

Garwood, N.C. 1985. Earthquake-caused landslides in Panama: Recovery of vegetation. Res. Rep. Natl. Geogr. Soc., (21): 181-184. 
Garwood, N.C.; D.P. Janos y N. Brokaw. 1979. Earthquake-caused landslides: A major disturbance in tropical forests. Science, (205): 997-999.

Gentry, A.H. 1988. Changes in plant communidady diversity and floristic composition on environmental and geographical gradients. Ann. Missouri Bot. Gard., (75): 1-34.

Gentry, A.H. 1995. Patterns of diversity and floristic composition in neotropical montane forests. En Churchill, S.P.; H. Balslev; E. Forero y J.L. Luteyn (Eds.): Biodiversity and conservation of Neotropical montane forests, pp. 103-126. Nueva York: NYBG.

Gentry, A.H. y C.H. Dodson 1987. Contribution of nontrees to species richness of a tropical rain forest. Biotropica, (19): 149-156.

Grubb, P.J.; J.R. Lloyd; T.D. Pennington y T.C. Whitmore. 1963. A comparison of montane and lowland rain forest in Ecuador - The forest structure, physiognomy, and floristics. J. Ecol., (51): 567-601.

Grubb, P.J. y T.C. Whitmore. 1966. A comparison of montane and lowland rain forest in Ecuador II. The climate and its effects on the distribution and physiognomy of the forests. J. Ecol., (54): 303-333.

Guariguata, M.R. 1990. Landslide disturbance and forest regeneration in the upper Luquillo mountains of Puerto Rico. J. Ecol., (78): 814-832.

Hall, M. 1977. El volcanismo en el Ecuador. Quito: Abya Yala.

Hamilton, L.S.; J.O. JUVIK y F.N. SCATENA (Eds.). 1994. Tropical Montane Cloud Forests. Ecological Studies 110: Nueva York: Springer

Hammen, T. van der; A. Pérez Precario y E.P. Pinto (Eds.). 1983. Studies on tropical Andean ecosystems, vol. 1. Vaduz: Cramer. 345 p.

Hammen, T. van der y P.M. RUIZ (Eds.). 1984. Studies on tropical Andean ecosystems, vol. 2. Vaduz: Cramer. 603 p.

Hammen, T. van der; S. Díaz-Piedrahita y V.J. Álvarez (Eds.). 1989. Studies on tropical Andean ecosystems, vol. 3. Vaduz: Cramer. 600 p.

Hammen, T. van der y A.G. Dos Santos (Eds.) 1995. Studies on tropical Andean ecosystems, vol. 4. Vaduz: Cramer. 603 p.

Hammen, T. van der; D. Mueller-Dombois y M.A. Little. 1989. Manual of Methods for Mountain Transect Studies. París: Unesco.

Herzog, T. 1923. Die Pflanzenwelt der bolivianischen Anden und ihres oestlichen. Leipzig: Vorlands.

Ibisch, P.L. 1996. Neotropische Epiphytendiversitaet - das Beispiel Bolivien. Archiv naturwissenschaftlicher Dissertationen 1. Wiehl.

Johansson, D.R. 1974. Ecology of vascular Epiphytes in west African rain forests. Acta Phytogeographica Suecia, (59): 1-136.
Joergensen, P.M. 1991. Species composition and present extension of Andean forest above 2,400 m altitude in Ecuador. Ph.D. thesis, Botanical Institute of Aarhus University.

Joergensen, P.M. y C. Ulloa Ulloa. 1994. Seed plants of the High Andes of Ecuador - a checklist. $A A U$ Reports 34: 1-443.

Joergensen, P.M. y S. León-Yanez (Eds.). 1999. Catalogue of the vascular plants of Ecuador. Monographs in Systematic Botany from the Missouri Botanical Garden 75.

Keating, P.L. 1995. Disturbance regimes and regeneration dynamics of Upper Montane Forests and Paramos in the Southern Ecuadorian Andes. $\mathrm{PhD}$ tesis, University of Colorado, Boulder.

Keating, P.L. 1997. An inventory of vascular plant species in the Páramo of Cajanuma, Podocarpus National Park (Ecuador). Phytologia, (83): 333-344.

Keating, P.L. 1998. Effects of anthropogenic disturbance on paramo vegetation in Podocarpus National Park, Ecuador. Physical Geography, (19): 221-238.

Keating, P.L. 1999. Changes in paramo vegetation along an elevational gradient in southern Ecuador. Journal of the Torrey Botanical Society, (126): 159-172.

Keating, P.L. 2000. Chronically disturbed paramo vegetation at a site in southern Ecuador. Journal of the Torrey Botanical Society, (127): 162-171.

Kessler, M. 1999. Plant species richness and endemism during natural landslide succession in a perhumid montane forest in the Bolivian Andes. Ecotropica, (5/2): 123-136.

Kitayama, K. 1992. An altitudinal transect study of the vegetation on Mount Kinabalu, Borneo. Vegetatio, (102): 149-171.

Leigh, E.G. 1999. Tropical forest ecology - a view from Barro Colordo Island. Oxford: Oxford University Press.

Madsen, J.E. 1989. Aspectos generales de la flora y vegetación del Parque Nacional Podocarpus. Boletín informativo sobre biología, conservación y vida silvestre, (1): 59-74.

Madsen, J.E. 1991. Floristic composition, structure and dynamics of an upper montane rain forest in Southern Ecuador. Ph.D. thesis, Botanical Institute of Aarhus University.

Madsen, J.E. \& B. OELLGAARD. 1994. Floristic composition, structure and dynamics of an upper montane rain forest in Southern Ecuador. Nord. J. Bot., (14/4): 403-423.

Meier, W. 1998. Flora und Vegetation des AvilaNationalparks Venezuela (Kuestenkordillere) unter besonderer Beruecksichtigung der Nebelwaldstufe. Dissertationes Botanicae 296. Berlín: Cramer.

Mueller-Dombois, D. \& H. Ellenberg 1974. Aims and methods of vegetation ecology. Nueva York: Wiley. 
Myers, N.; R.A. Mittermeier; C.G. Mittermaier; G.A.B. DA Fonseca y J. Kent. 2000. Biodiversity hotspots for conservation priorities. Nature, (403/25): 853-858.

Oellgaard, B. y J.E. Madsen. 1993. Inventario preliminar de las especies vegetales en el Parque Nacional Podocarpus. Revista de Difusión Técnica y Científica de la Facultad de Ciencias Agrícolas, Universidad Nacional de Loja, (2223 1/2): 66-87.

Paulsch, A y C. Czimczik. 2001. Classification of tropical mountain shrub vegetation - a structural approach. Die ERDE, (132): 27-41.

Schrumpf, M.; G. Guggenberger; C. Valarezo y W. Zech. 2001. Tropical rain forest soils. Die ERDE, (132): 43-59.

Sierra, R. (Ed.). 1999. Propuesta preliminar de un sistema de clasificación de vegetación para el Ecuador Continental. Quito: GEF. 194 p.
Simonett, D.S. 1967. Landslide distribution and earthquakes in the Bewani and Torricelli mountains, New Guinea. En: Jennings, J.N. y J.A. Mabbutt (Eds.): Landform studies from Australia and New Guinea, pp. 64-84, Canberra.

Stern, M.J. 1995. Vegetation recovery on earthquaketriggered landslide sites in the Ecuadorian Andes. En: Churchill, S.P.; H. Balslev; E. Forero y J.L. Luteyn (Eds.): Biodiversity and conservation of neotropical montane forests, 207-220. Nueva York: NYBG.

Ulloa, C. y P.M. Joergenson. 1993. Árboles y arbustos de los Andes del Ecuador. AAU Reports, (30): 1-263.

Webster, G.L. 1995. The Panorama of Neotropical Cloud Forest. En Churchill, S.P.; H. Balslev; E. Forero y J.L. Luteyn (Eds.): Biodiversity and conservation of neotropical montane forests, 53-78. Nueva York: NYBG. 